\title{
Plasma circulating-microRNA profiles are useful for assessing prognosis in patients with cytogenetically normal myelodysplastic syndromes
}

\author{
Zhuang Zuo ${ }^{1,5}$, Sourindra Maiti ${ }^{2,5}$, Shimin $\mathrm{Hu}^{1}$, Sanam Loghavi ${ }^{1}$, George A Calin ${ }^{3}$, \\ Guillermo Garcia-Manero ${ }^{4}$, Hagop M Kantarjian ${ }^{4}$, L Jeffrey Medeiros ${ }^{1}$, \\ Laurence JN Cooper ${ }^{2,6}$ and Carlos E Bueso-Ramos ${ }^{1,6}$
}

${ }^{1}$ Department of Hematopathology, The University of Texas MD Anderson Cancer Center, Houston, TX, USA; ${ }^{2}$ Department of Pediatrics, The University of Texas MD Anderson Cancer Center, Houston, TX, USA; ${ }^{3}$ Department of Experimental Therapeutics, The University of Texas MD Anderson Cancer Center, Houston, TX, USA and ${ }^{4}$ Department of Leukemia, The University of Texas MD Anderson Cancer Center, Houston, TX, USA

\begin{abstract}
Myelodysplastic syndromes are a heterogeneous group of clonal bone marrow hematopoietic stem cell disorders characterized by ineffective hematopoiesis and peripheral cytopenias. Chromosomal abnormalities and gene mutations have been shown to have essential roles in pathogenesis and correlate with prognosis. Molecular markers, however, are not integrated into currently used prognostic systems. The goal of this study is to identify plasma microRNAs useful for classification and risk stratification of myelodysplastic syndromes. We applied a novel, high-throughput digital quantification technology (NanoString) to profile microRNA expression in plasma samples of 72 patients with myelodysplastic syndromes and 12 healthy individuals. We correlated these results with overall survival. In patients with myelodysplastic syndromes associated with a diploid karyotype, we identified and validated a 7-microRNA signature as an independent predictor of survival with a predictive power of $75 \%$ accuracy $(P=0.008)$, better than those of the International Prognostic Scoring Systems and the MD Anderson Prognostic Lower Risk Prognostic Model. We also identified differentially expressed plasma microRNAs in patients with myelodysplastic syndromes versus healthy individuals and between patients with myelodysplastic syndromes associated with different cytogenetic features. These results validate the utility of circulating-microRNA levels as noninvasive biomarkers that can inform the management of patients with myelodysplastic syndromes. Our findings also shed light on interactions of gene regulation pathways that are likely involved in the pathogenesis of myelodysplastic syndromes.

Modern Pathology (2015) 28, 373-382; doi:10.1038/modpathol.2014.108; published online 12 September 2014
\end{abstract}

Myelodysplastic syndromes are a group of clonal bone marrow stem cell disorders that mostly affect the elderly. In the United States, 10000 to 20000 patients with myelodysplastic syndromes are newly diagnosed each year. ${ }^{1}$ Individuals with

Correspondence: Dr Z Zuo, MD, PhD, Department of Hematopathology, Unit 149, The University of Texas MD Anderson Cancer Center, 1515 Holcombe Boulevard, Houston, TX 77030, USA.

E-mail: zzuo@mdanderson.org

${ }^{5}$ These authors contributed equally to this work.

${ }^{6}$ These authors contributed equally to this work.

Received 8 April 2014; revised 24 June 2014; accepted 25 June 2014; published online 12 September 2014 myelodysplastic syndromes exhibit blood cytopenias, ineffective hematopoiesis, usually hypercellular or normocellular bone marrow, and have a high risk of transformation into acute myeloid leukemia in a subset of cases. The survival rate of patients with myelodysplastic syndromes varies greatly, from a few months for aggressive diseases to a near-normal life expectancy for indolent diseases. Thus, it is important to stratify patients with myelodysplastic syndromes into low- or highrisk groups for optimal patient management.

Until recently, systems for predicting the outcome of patients with myelodysplastic syndromes have been based on clinicopathologic and cytogenetic criteria, such as age, bone marrow blast percentage, 
karyotype, and peripheral blood cytopenias. The International Prognostic Scoring System is the most widely used system for assessment of prognosis and planning of therapy. ${ }^{2-4}$ Among all parameters included in this model, conventional cytogenetic analysis has been shown to be the strongest predictor of prognosis. Patients with complex cytogenetic abnormalities, or certain single abnormalities such as $+8, \operatorname{del}(7 q)$, or -7 , are considered to have an intermediate or poor prognosis, whereas patients with other single cytogenetic abnormalities, such as $\operatorname{del}(5 q)$, del(20q), or $-Y$, are considered to have a good or very good prognosis. ${ }^{5}$ Nevertheless, over half of the patients with myelodysplastic syndromes do not have detectable cytogenetic abnormalities (cytogenetically normal with a diploid karyotype) and are included in the good-prognostic group. ${ }^{6}$ This obviously limits the predictive power of the model for patients with myelodysplastic syndromes.

Many of the challenges to improving risk stratification and prognostic assessment of patients with myelodysplastic syndromes can be attributed to our limited understanding of the mechanisms that lead to the development and progression of these diseases. The onset of myelodysplastic syndromes and its transformation into acute myeloid leukemia is believed to be a multi-step process requiring the accumulation of genetic and epigenetic alterations. ${ }^{7,8}$ Alterations in apoptosis and proliferation have been implicated in the pathogenesis of myelodysplastic syndromes, and recurrent gene mutations have been associated with the disease progression and outcome. ${ }^{9,10}$ In addition, gene expression studies have identified markers that appear to improve the accuracy of risk stratification or prognostication; ${ }^{11}$ however, none of these markers have been included in the original or revised International Prognostic Scoring System. ${ }^{12}$

MicroRNAs are a class of short (19-25 nucleotides), single-stranded RNA molecules that are components of the epigenetic machinery. ${ }^{13}$ MicroRNAs regulate the expression of target genes post-transcriptionally, mostly by inhibiting translation or inducing mRNA degradation. MicroRNAs also have important roles in the regulation of DNA methylation and histone modification, and can function as oncogenes, tumor suppressor genes, or both. MicroRNAs have been implicated specifically in the development of solid tumors and hematopoietic malignancies. ${ }^{14}$ Substantial levels of microRNAs have been detected in several body fluids, such as plasma, serum, urine, breast milk, and saliva. ${ }^{15}$ Specific circulating-microRNA signatures have been identified that correlate with the diagnosis or outcome of various diseases, especially cancers. ${ }^{15-20}$ Therefore, circulating microRNAs have the potential to be used as noninvasive biomarkers for cancer diagnosis and prognosis.

We recently reported that plasma levels of let-7a and miR-16 can predict the survival of patients with myelodysplastic syndromes more accurately than the International Prognostic Scoring System. ${ }^{21}$
In the current study, we expanded our efforts to globally profile plasma microRNA expression patterns in patients with myelodysplastic syndromes and correlated these results with specific cytogenetic features. We identified and validated robust circulating-microRNA signatures that are helpful for the diagnosis of myelodysplastic syndromes and are also useful for assessment of prognosis in these patients.

\section{Materials and methods}

\section{Patient Samples}

The study cohort included a subset of patients with myelodysplastic syndromes who were seen at The University of Texas MD Anderson Cancer Center between 2006 and 2008. For each patient, pretreatment plasma samples were analyzed and clinical follow-up of more than 6 months was available (unless the patient died of the disease within 6 months). The pathologic diagnosis of each case of myelodysplastic syndrome was based on criteria defined by the 2008 World Health Organization (WHO) classification. ${ }^{3}$

For this study we used strict criteria for case selection: (1) we included only patients with de novo myelodysplastic syndromes who had no known history of malignancy, chemotherapy, or radiation therapy; (2) we focused mainly on patients with cytogenetically normal myelodysplastic syndromes, with only a small number of cases with single abnormalities of $\operatorname{del}(7 q) /-7$ or $\operatorname{del}(20 q)$; (3) to minimize the influence of known myelodysplastic syndrome-related gene mutations, we excluded all patients who tested positive for common mutations in the FLT3, NPM1, RAS, IDH1, IDH2, and KIT genes; and (4) we excluded patients who had undergone hematopoietic stem cell transplant. The final study cohort consisted of 72 patients with de novo myelodysplastic syndromes that satisfied our selection criteria. We also included plasma samples from 12 healthy individuals who were random donors with normal complete blood count. Plasma was obtained by standard venipuncture and centrifugation in EDTA-coated tubes, and then frozen at $-80{ }^{\circ} \mathrm{C}$ prior to analysis. Written informed consent was obtained from all participants in accordance with the Declaration of Helsinki, and the study was approved by the institutional review board.

\section{RNA Extraction from Plasma Samples and microRNA Expression Profiling}

RNA was extracted from $500 \mu \mathrm{l}$ of plasma obtained from each patient using the Norgen Plasma/Serum Circulating and Exosomal RNA Purification Kit (Norgen Biotek, Thorold, Ontario, Canada) according to the manufacturer's protocol. RNA was eluted in $100 \mu \mathrm{l}$ water and concentrated to $20 \mu \mathrm{l} ; 3 \mu \mathrm{l}$ was 
used for profiling on the multiplexed nCounter platform from NanoString Technologies (Seattle, WA, USA).

The nCounter microRNA Assay detects simultaneously 800 human microRNAs in each sample. RNA samples were prepared by ligating a specific DNA tag (miR-tag) onto the $3^{\prime}$ end of each mature microRNA according to the manufacturer's instructions. Excess tags were removed by restriction digestion at $37^{\circ} \mathrm{C}$. Hybridizations were carried out by combining $5 \mu \mathrm{l}$ of each microRNA-miR-Tag sample with $20 \mu \mathrm{l}$ of nCounter Reporter probes in hybridization buffer and $5 \mu \mathrm{l}$ of nCounter Capture probes (for a total reaction volume of $30 \mu \mathrm{l}$ ) at $65{ }^{\circ} \mathrm{C}$ for 16-20 h. Excess probes were removed using a twostep magnetic bead-based purification on the nCounter Prep Station. Abundance of specific target molecules was quantified using the nCounter Digital Analyzer by counting the individual fluorescent barcodes and assessing target molecules. The data were collected using the nCounter Digital Analyzer after taking images of the immobilized fluorescent reporters in the sample cartridge using a CCD camera.

\section{Data Analysis}

MicroRNA data were analyzed using the nSolver software (NanoString Technologies) and SPSS software from IBM (Armonk, NY, USA). Batch effects were eliminated using ComBat, an empirical Bayes method. ${ }^{22}$ The level of each microRNA was normalized to the total count of the entire measured microRNA content in each sample. ANOVA was performed with a cutoff $P$-value of 0.05 to identify a set of microRNAs that had the highest differences in means across samples. Patient survival was plotted by the Kaplan-Meier method and differences were compared using the log rank test. Multivariate analysis was performed by Cox proportional regression model. To compare the overall survival of patients with cytogenetically normal myelodysplastic syndromes, we used 30 months of overall survival as a cutoff to divide the patients into goodand poor-survival groups. This cutoff correlates with the reported overall survival of the International Prognostic Scoring System low patients. ${ }^{23}$ A support vector machines model was applied to assess the predictive power of the gene signature (genepattern. broadinstitute.org). The discriminative abilities of the support vector machines models were determined according to receiver operating characteristic curve analysis.

\section{Results}

\section{Patient Characteristics}

The clinicopathologic characteristics of the 72 patients with myelodysplastic syndromes in the study group are summarized in Table 1 . These
Table 1 Clinicopathologic characteristics of the patients with myelodysplastic syndromes in this study

Characteristics

Age, median (range), years

$67(36-87)$

Sex

Male

Female

WHO classification

Refractory cytopenia with unilineage dysplasia

Refractory anemia with ring sideroblasts

Refractory cytopenia with multilineage dysplasia

Refractory anemia with excess blasts -1

Refractory anemia with excess blasts -2

42

Cytogenetic features

Diploid

Isolated $\operatorname{del}(7 q) /-7$

Isolated $\operatorname{del}(20 \mathrm{q})$

International Prognostic Scoring System risk score Low

Intermediate-1

Intermediate-2

High

patients had a median age of 67 years (range, 36-87 years), and there were 42 women and 30 men. According to the 2008 WHO classification, the pathologic diagnoses for these myelodysplastic syndrome cases were refractory cytopenia with unilineage dysplasia $(n=12)$, refractory anemia with ring sideroblasts $(n=7)$, refractory cytopenia with multilineage dysplasia $(n=28)$, refractory anemia with excess blasts-1 $(n=14)$, and refractory anemia with excess blasts-2 $(n=11)$. Cytogenetically, 47 patients had a diploid karyotype, 13 patients had isolated $\operatorname{del}(7 q)$ or monosomy7, and 12 had isolated del(20q). These patients were also stratified into four risk groups according to their International Prognostic Scoring System risk score: 24 low, 29 intermediate-1, 18 intermediate-2, and 1 high.

\section{Identification and Validation of a Prognostic Plasma microRNA Signature}

The main purpose of this study was to identify plasma microRNAs that can serve as prognostic biomarkers in patients with cytogenetically normal myelodysplastic syndromes with no other risk factors. Therefore, we randomly selected 35 patients with cytogenetically normal myelodysplastic syndromes as the training set. Among 800 human microRNAs assessed in each nCounter microRNA assay, 639 microRNAs consistently generated analyzable signals in all samples. Hence, these 639 microRNAs were used in normalization and comparison analyses. To identify plasma microRNAs that correlated with survival in patients with cytogenetically normal myelodysplastic syndromes, we used an overall survival of 30 months as a cutoff 
Table 2 Seven most differentially expressed plasma microRNAs between good- and poor-survival groups of patients with cytogenetically normal myelodysplastic syndromes

\begin{tabular}{lccc}
\hline & \multicolumn{2}{c}{ Expression (mean \pm s.d.) } & \\
\cline { 2 - 3 } & Poor survival & Good survival & \\
\hline hsa-let-7a & $2.85 \pm 1.79$ & $1.17 \pm 1.5$ & 0.005 \\
hsa-miR-144 & $6.13 \pm 2.83$ & $4.26 \pm 2.23$ & 0.036 \\
hsa-miR-16 & $43.42 \pm 18.22$ & $-2.35 \pm 47.66$ & $<0.001$ \\
hsa-miR-25 & $15.58 \pm 6.27$ & $2.8 \pm 15$ & $<0.001$ \\
hsa-miR-451 & $169.55 \pm 72.79$ & $39.07 \pm 65.18$ & $<0.001$ \\
hsa-miR-651 & $1.03 \pm 0.5$ & $2.44 \pm 1.18$ & $<0.001$ \\
hsa-miR-655 & $0.67 \pm 0.35$ & $1.52 \pm 0.66$ & $<0.001$ \\
\hline
\end{tabular}

to divide the 35 patients with cytogeneticallynormal-myelodysplastic syndromes of the training set into good- and poor-survival groups (for reasons explained in the methods). Comparing the expression profiles, we identified seven highly differentially expressed microRNAs that correlated with patient survival (Table 2). Unsupervised hierarchical clustering showed that these seven microRNAs separated all patients into two main groups (Figure 1a). Kaplan-Meier plots of the overall survival between these two patient groups was significant (Figure $1 \mathrm{~b} ; P=0.014$ ). Multivariate analysis indicated that this seven-microRNA signature predicts overall survival independently of patient age, gender, and the International Prognostic Scoring System score with a hazard ratio of 6.54 (95\% CI: 1.64-26.17; $P=0.008$ ).

To validate the seven-microRNA signature, we profiled microRNA expression in plasma samples from an independent set of 12 patients with cytogenetically normal myelodysplastic syndromes. These patients were also divided into good- or poor-survival groups based on a cutoff of 30 months for overall survival. When applying the sevenmicroRNA signature to predict the overall survival of these validation cases using the support vector machines method, the prediction accuracy was $75 \%$ (Table 3). In contrast, using the International Prognostic Scoring System to predict patient overall survival, with International Prognostic Scoring System low indicating good survival and International Prognostic Scoring System Intermediate-1 or Intermediate-2 indicating poorer survival, the prediction accuracy was $50 \%$ (Table 3). Less accurate predictions resulted from applying the Revised International Prognostic Scoring System or the MD Anderson Prognostic Lower Risk Prognostic Model. ${ }^{24}$

\section{Plasma microRNA Expression Patterns in Cytogenetically Abnormal Myelodysplastic Syndromes and Healthy Individuals}

To further explore whether there are common plasma microRNA expression patterns in patients with myelodysplastic syndromes, we also profiled plasma samples from 14 patients with isolated $\operatorname{del}(7 q) /-7$, a known poor-prognostic group, and 11 patients with isolated del(20q), a known goodprognostic group, as well as those from 12 healthy individuals. These results were pooled with those from the training set and reanalyzed together. After normalization, the top 30 differentially expressed microRNAs between patients with myelodysplastic syndromes and healthy individuals are listed in Table 4. Unsupervised hierarchical clustering of all 84 cases with these 30 microRNAs revealed distinct expression patterns between patients with myelodysplastic syndromes patients and healthy individuals (Figure 1c).

We then sought to identify specific plasma microRNA expression patterns that could distinguish patients with myelodysplastic syndromes associated with isolated $\operatorname{del}(7 q) /-7$ or $\operatorname{del}(20 q)$, and might relate to the specific chromosomal alterations. Compared with the remaining myelodysplastic syndrome cases, levels of eight microRNAs were found to be significantly different in patients with myelodysplastic syndrome associated with isolated $\operatorname{del}(7 q) /-7$ (Table 5). The genetic location of four of these differentially expressed microRNAs has been mapped to chromosome 7 , including miR-96, miR-196b, miR-25, and miR-590. Plasma levels of all of these microRNAs, except miR-96, were significantly lower in patients with myelodysplastic syndrome associated with isolated $\operatorname{del}(7 q) /-7$. For patients with myelodysplastic syndromes associated with isolated $\operatorname{del}(20 \mathrm{q})$, the plasma levels of 13 microRNAs were found to be significantly different from those of the remaining patients with myelodysplastic syndromes (Table 5). Two of these microRNAs, miR-296 and miR-499, are located on the long arm of chromosome 20, but their plasma levels were significantly higher in this subgroup of patients. We were unable to identify a set of microRNAs that effectively separated patients with $\operatorname{del}(7 q) /-7$ or $\operatorname{del}(20 q)$ from patients with other subtypes of myelodysplastic syndromes, indicating the heterogeneity within these cytogenetic subgroups.

Putting these findings together, we observed that several microRNAs were repeatedly significant during these comparisons and there seemed to be some consistent patterns as listed in Table 6. Mostly, miR-144 and miR-451 were upregulated in patients with myelodysplastic syndromes when compared with healthy individuals. MiR-144 and miR-451 levels were further upregulated in myelodysplastic syndromes associated with isolated $\operatorname{del}(7 q) /-7$, which represented a poor-prognostic group, and were relatively downregulated in myelodysplastic syndromes associated with isolated del(20q), a good-prognostic group. Among patients with a diploid karyotype, higher levels of these microRNAs were also seen in patients with a poor overall survival, as reflected in the 7-microRNA signature. 
a

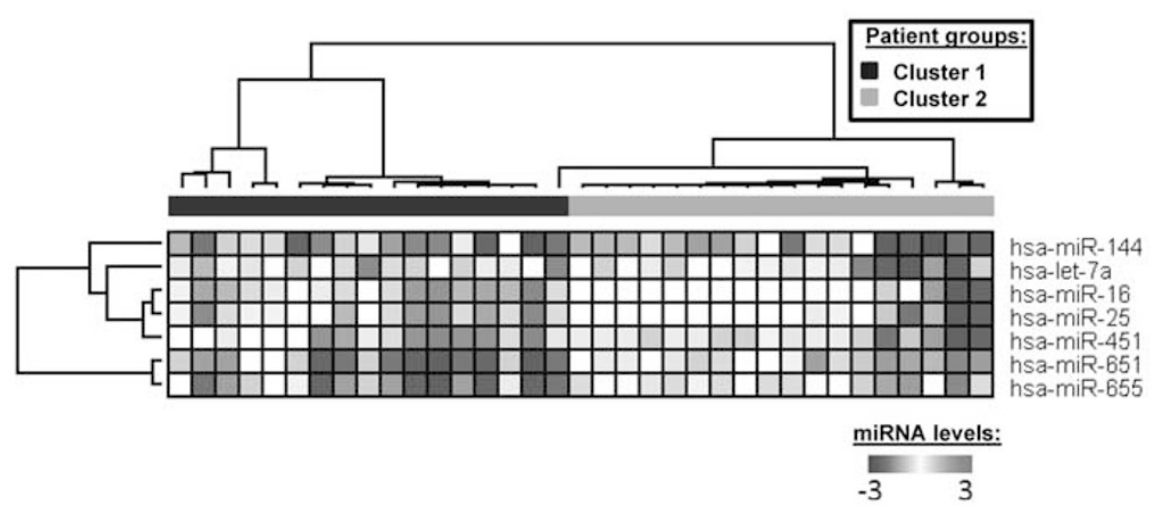

b

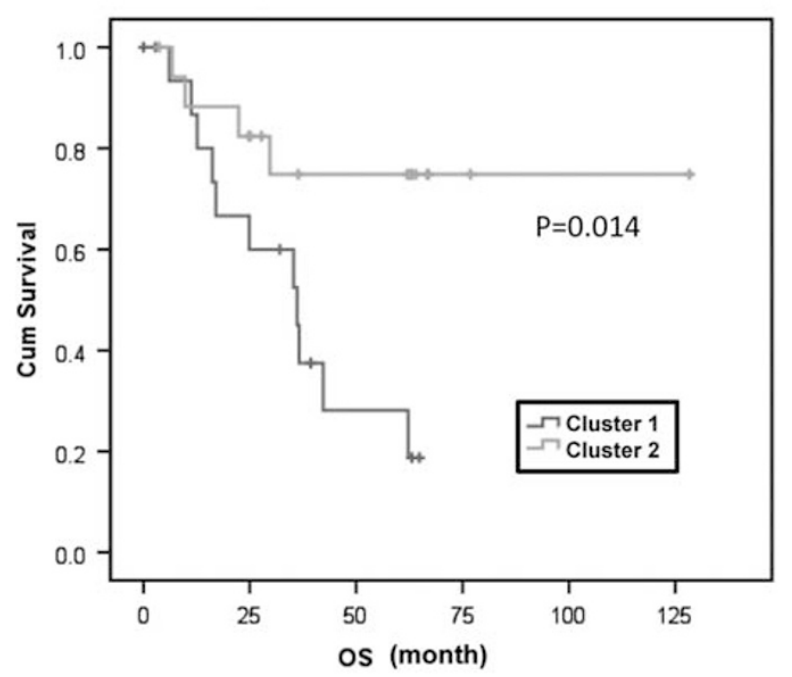

C

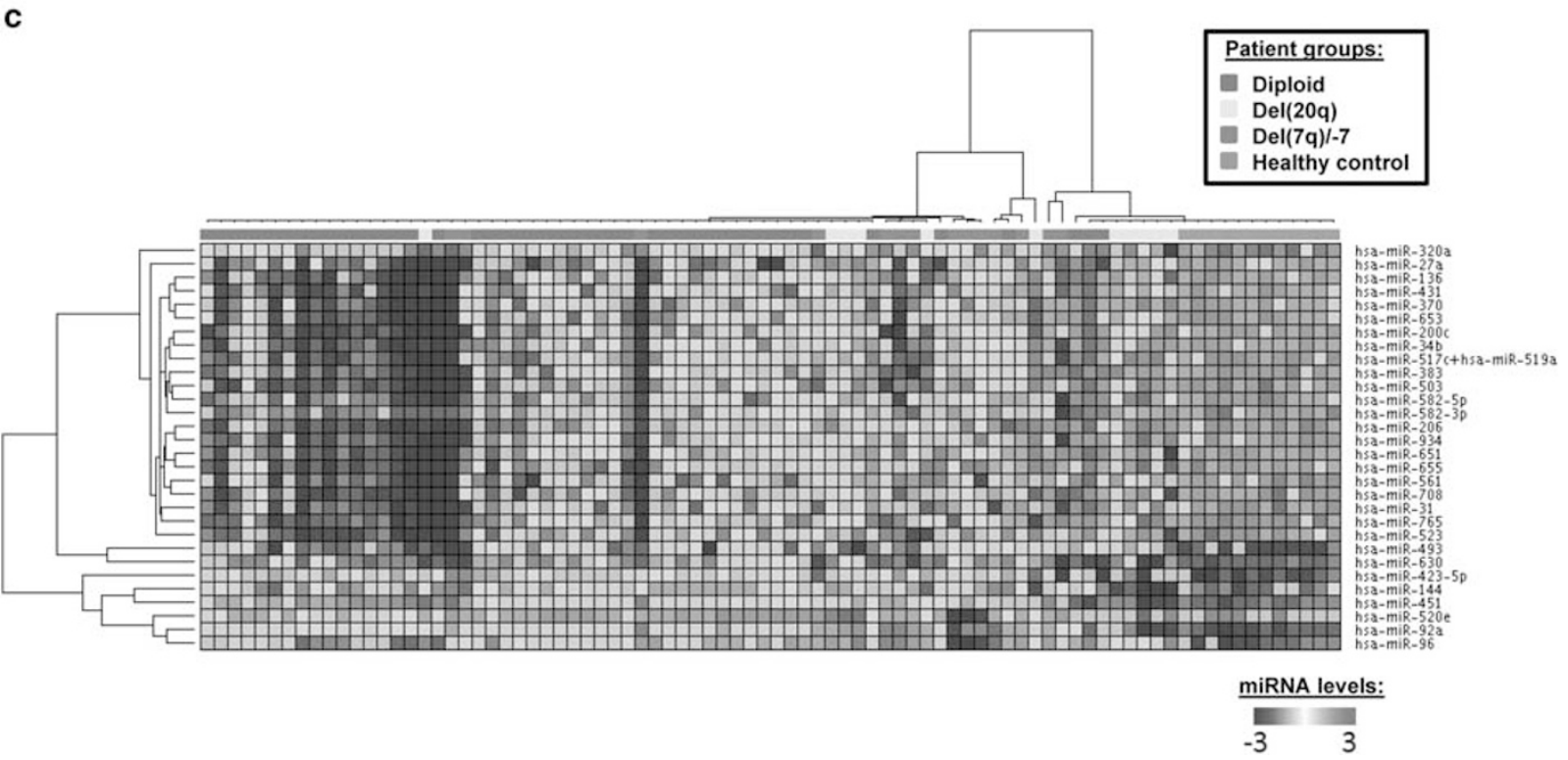

Figure 1 The expression patterns of plasma microRNAs are associated with myelodysplastic syndromes subtypes and patient survival. (a) Unsupervised hierarchical clustering using a seven-microRNA signature separates 35 cytogenetically normal myelodysplastic syndrome patients into two clusters. (b) Overall survival of patients in these two clusters from the hierarchical clustering differ significantly $(P=0.014)$. (c) Unsupervised hierarchical clustering using the top 30 differentially expressed plasma microRNAs distinguishes myelodysplastic syndrome patients from healthy individuals. 
Similar patterns were also observed for miR-206, miR-34b, miR-503, miR-651, miR-655, miR-92a, and miR-96. Expression patterns of miR-25 and miR-493, by contrast, were not consistently correlated with clinical or pathological features.

Table 3 Predicting overall survival in 12 patients with cytogenetically normal myelodysplastic syndromes using the 7-microRNA signature and various prognostic models

\begin{tabular}{lcc}
\hline & \multicolumn{2}{c}{ True survival } \\
\cline { 2 - 3 } & Poor & Good \\
\hline Predicted survival by miRNAs & & \\
Poor & $75 \%(6)$ & $25 \%(1)$ \\
Good & $25 \%(2)$ & $75 \%(3)$ \\
Predicted survival by International Prognostic Scoring System \\
Intermediate-1/2 & $50 \%(4)$ & $50 \%(2)$ \\
Low & $50 \%(4)$ & $50 \%(2)$ \\
& & \\
Predicted survival by Revised International Prognostic Scoring \\
System & $37.5 \%(3)$ & $50 \%(2)$ \\
Intermediate/high & $62.5 \%(5)$ & $50 \%(2)$ \\
Low/very low & \multicolumn{2}{c}{} \\
Predicted survival by MD Anderson Prognostic Score \\
Category 2/3 & $37.5 \%(3)$ & $100 \%(4)$ \\
Category 1 & $62.5 \%(5)$ & $0 \%(0)$ \\
\hline
\end{tabular}

\section{Discussion}

In an earlier study, we reported that plasma microRNA levels have predictive value for assessing the prognosis of patients with myelodysplastic syndromes. ${ }^{21}$ However, several issues remain to be addressed. A global plasma microRNA expression profile in patients with myelodysplastic syndromes is still unknown. Given the high heterogeneity of myelodysplastic syndromes, finding optimal biomarkers for specific subgroups of patients is required for microRNA profiling to have clinical applications. Technically, the absence of reliable normalizers for assessing plasma microRNAs individually by PCR also limits clinical utilization. In this study, our aim was to expand previous efforts by identifying plasma microRNA signatures that could improve the prognostication of patients with cytogenetically normal myelodysplastic syndromes with no other known risk factors. Our results suggest that plasma microRNA signatures can predict prognosis in patients with myelodysplastic syndromes better than the currently used prognostic scoring systems.

We used the highly sensitive nCounter platform ${ }^{25}$ to assess the global microRNA expression patterns in plasma of patients with myelodysplastic syndromes. Global microRNA expression profiling has major advantages over quantitative real-time PCR.

Table 4 Top 30 plasma microRNAs differentially expressed between patients with myelodysplastic syndromes and healthy individuals

\begin{tabular}{|c|c|c|c|}
\hline & Upregulated in & Fold change & $\mathrm{P}$ \\
\hline hsa-miR-136 & Healthy & 2.02 & $<0.001$ \\
\hline hsa-miR-144 & Myelodysplastic syndromes & 2.76 & $<0.001$ \\
\hline hsa-miR-200c & Healthy & 2.03 & $<0.001$ \\
\hline hsa-miR-206 & Healthy & 2.06 & $<0.001$ \\
\hline hsa-miR-27a & Healthy & 2.01 & $<0.001$ \\
\hline hsa-miR-31 & Healthy & 2.23 & $<0.001$ \\
\hline hsa-miR-320a & Healthy & 2.36 & $<0.001$ \\
\hline hsa-miR-34b & Healthy & 1.88 & $<0.001$ \\
\hline hsa-miR-370 & Healthy & 1.91 & $<0.001$ \\
\hline hsa-miR-383 & Healthy & 2.32 & $<0.001$ \\
\hline hsa-miR-423-5p & Myelodysplastic syndromes & 5.21 & $<0.001$ \\
\hline hsa-miR-431 & Healthy & 1.93 & $<0.001$ \\
\hline hsa-miR-451 & Myelodysplastic syndromes & 1.76 & $<0.001$ \\
\hline hsa-miR-493 & Myelodysplastic syndromes & 3.26 & $<0.001$ \\
\hline hsa-miR-503 & Healthy & 2.37 & $<0.001$ \\
\hline hsa-miR-517c + hsa-miR-519a & Healthy & 2.11 & $<0.001$ \\
\hline hsa-miR-520e & Myelodysplastic syndromes & 1.98 & $<0.001$ \\
\hline hsa-miR-523 & Healthy & 2.31 & $<0.001$ \\
\hline hsa-miR-561 & Healthy & 2.16 & $<0.001$ \\
\hline hsa-miR-582-3p & Healthy & 2.04 & $<0.001$ \\
\hline hsa-miR-582-5p & Healthy & 2.08 & $<0.001$ \\
\hline hsa-miR-630 & Myelodysplastic syndromes & 4.29 & $<0.001$ \\
\hline hsa-miR-651 & Healthy & 1.75 & $<0.001$ \\
\hline hsa-miR-653 & Healthy & 1.93 & $<0.001$ \\
\hline hsa-miR-655 & Healthy & 2.07 & $<0.001$ \\
\hline hsa-miR-708 & Healthy & 2.25 & $<0.001$ \\
\hline hsa-miR-765 & Healthy & 2.37 & $<0.001$ \\
\hline hsa-miR-92a & Myelodysplastic syndromes & 2.39 & $<0.001$ \\
\hline hsa-miR-934 & Healthy & 2.22 & $<0.001$ \\
\hline hsa-miR-96 & Myelodysplastic syndromes & 3.63 & $<0.001$ \\
\hline
\end{tabular}


Table 5 Differentially expressed plasma microRNAs in patients with myelodysplastic syndromes associated with either isolated $\operatorname{del}(7 q) /-7$ or isolated $\operatorname{del}(20 q)$

\begin{tabular}{|c|c|c|c|c|}
\hline & Up/downregulated & Fold change & $\mathrm{P}$ & Chromosomal location \\
\hline \multicolumn{5}{|c|}{$\operatorname{Del}(7 q) /-7$ vs the rest myelodysplastic syndromes } \\
\hline hsa-miR-140-5p & Down & 1.93 & 0.001 & $16 q 22.1$ \\
\hline hsa-miR-144 & Up & 1.63 & 0.027 & $17 \mathrm{q} 11.2$ \\
\hline hsa-miR-196b & Down & 1.56 & 0.026 & $7 \mathrm{p} 15.2$ \\
\hline hsa-miR-25 & Down & 1.5 & 0.025 & $7 \mathrm{q} 22.1$ \\
\hline hsa-miR-451 & $\mathrm{Up}$ & 1.67 & 0.031 & $17 q 11.2$ \\
\hline hsa-miR-590-3p & Down & 1.98 & 0.007 & $7 q 11.23$ \\
\hline hsa-miR-92a & $\mathrm{Up}$ & 2.22 & 0.021 & $13 q 31.3$ \\
\hline hsa-miR-96 & Up & 1.36 & 0.023 & $7 q 32.2$ \\
\hline \multicolumn{5}{|c|}{ Del(20q) vs the rest myelodysplastic syndromes } \\
\hline hsa-miR-144 & Down & 2.34 & 0.028 & $17 q 11.2$ \\
\hline hsa-miR-206 & Up & 1.55 & 0.018 & $6 \mathrm{p} 12.2$ \\
\hline hsa-miR-296-5p & Up & 1.91 & 0.021 & $20 q 13.32$ \\
\hline hsa-miR-323-5p & Up & 1.75 & 0.01 & $14 q 32.31$ \\
\hline hsa-miR-34b & Up & 1.5 & 0.02 & $11 \mathrm{q} 23.1$ \\
\hline hsa-miR-451 & Down & 4.95 & 0.01 & $17 \mathrm{q} 11.2$ \\
\hline hsa-miR-493 & Up & 2.36 & 0.038 & $14 q 32.2$ \\
\hline hsa-miR-499-5p & Up & 1.65 & 0.035 & $20 q 11.22$ \\
\hline hsa-miR-503 & Up & 1.52 & 0.049 & Xq26.3 \\
\hline hsa-miR-632 & Up & 1.67 & 0.01 & $17 q 11.2$ \\
\hline hsa-miR-769-5p & Up & 1.75 & 0.007 & $19 q 13.32$ \\
\hline hsa-miR-92a & Down & 2.99 & 0.038 & $13 q 31.3$ \\
\hline hsa-miR-98 & $\mathrm{Up}$ & 1.74 & 0.004 & Xp11.22 \\
\hline
\end{tabular}

Since a global picture of all microRNA species in plasma is obtained in every measurement, normalization to the entire content will more likely filter out the effects caused by the random fluctuations of individual microRNA levels. In contrast, microRNA levels assessed by quantitative real-time PCR are usually normalized to one or a small set of specific, so-called stably expressed microRNAs. No microRNA, however, has been shown consistently to be a good stable normalizer in plasma or serum for measuring individual microRNA levels. ${ }^{26}$

Since patients with myelodysplastic syndromes present with highly diverse clinicopathologic, cytogenetic, and genetic features that contribute to patient outcome, we attempted to select a more uniform patient cohort for this study. We therefore selected 72 patients with de novo myelodysplastic syndromes, most of whom had a diploid karyotype. For comparison, we also selected a small subset of patients with myelodysplastic syndromes associated with either isolated $\operatorname{del}(7 q) /-7$ or isolated $\operatorname{del}(20 q)$, representing two common types of myelodysplastic syndromes associated with a poor or good prognosis, respectively. To avoid confounding variables that could complicate analysis, we also excluded patients who had FLT3, NPM1, RAS, IDH1, IDH2, or KIT mutations, which in combination account for no more than $25 \%$ of cytogenetically normal cases of myelodysplastic syndrome. ${ }^{6,9}$ Mutations in other myelodysplastic syndrome-related genes, especially TET2, ASXL1, TP53, and EZH2, were not tested in this study. ${ }^{6,10}$

Using global profiling, we identified a list of differentially expressed plasma microRNAs in patients with myelodysplastic syndromes with different outcomes, among patients with different cytogenetic features, and between patients with myelodysplastic syndromes versus healthy individuals. For patients with a diploid karyotype and none of the common myelodysplastic syndromerelated mutations, admittedly a heterogeneous group, there are no known clinicopathologic or biologic markers that can further stratify these patients. Our seven-microRNA signature showed $75 \%$ accuracy in predicting survival in patients with diploid myelodysplastic syndromes. This signature also included two microRNAs, let-7a and miR-16, that we identified previously. ${ }^{21}$ We also identified subsets of microRNAs that are expressed differentially in myelodysplastic syndromes associated with isolated $\operatorname{del}(7 q) /-7$ or isolated $\operatorname{del}(20 q)$, respectively. We observed consistent expression patterns of several microRNAs that distinguish patients with myelodysplastic syndromes from healthy individuals, and between myelodysplastic syndromes associated with good or poor prognosis. A good example is the miR-144 and miR-451 pair, whose levels were consistently low in healthy individuals, and in patients with diploid myelodysplastic syndromes associated with good overall survival or patients with isolated del(20q). In contrast, levels of miR-144 and miR-451 were high in diploid patients with poor overall survival and in patients with isolated $\operatorname{del}(7 q) /-7$.

There is a biological rationale for miR-144 and miR-451 having predictive value in patients with myelodysplastic syndromes. Both of these microRNAs are encoded by the vertebrate-specific 
Table 6 Levels of plasma microRNAs comparing different patient subsets

\begin{tabular}{|c|c|c|c|c|}
\hline & $\begin{array}{l}\text { Healthy individual vs } \\
\text { myelodysplastic syndromes }\end{array}$ & $\begin{array}{l}\text { Del(20q) vs other } \\
\text { myelodysplastic syndromes }\end{array}$ & $\begin{array}{l}\operatorname{Del}(7 q) /-7 \text { vs other } \\
\text { myelodysplastic syndromes }\end{array}$ & $\begin{array}{c}\text { Poor overall survival vs good } \\
\text { overall survival }\end{array}$ \\
\hline hsa-miR-144 & $\downarrow$ & $\downarrow$ & $\uparrow$ & $\uparrow$ \\
\hline hsa-miR-206 & $\uparrow$ & $\uparrow$ & - & - \\
\hline hsa-miR-25 & $\downarrow$ & - & $\downarrow$ & $\uparrow$ \\
\hline hsa-miR-34b & $\uparrow$ & $\uparrow$ & - & - \\
\hline hsa-miR-451 & $\downarrow$ & $\downarrow$ & $\uparrow$ & $\uparrow$ \\
\hline hsa-miR-493 & $\downarrow$ & $\uparrow$ & - & - \\
\hline hsa-miR-503 & $\uparrow$ & $\uparrow$ & - & - \\
\hline hsa-miR-651 & $\uparrow$ & - & - & $\downarrow$ \\
\hline hsa-miR-655 & $\uparrow$ & - & - & $\downarrow$ \\
\hline hsa-miR-92a & $\downarrow$ & $\downarrow$ & $\uparrow$ & - \\
\hline hsa-miR-96 & $\downarrow$ & - & $\uparrow$ & - \\
\hline
\end{tabular}

Symbols: $\uparrow$, upregulated; $\downarrow$, downregulated; —, no significant difference.

miR-144/451 cluster on chromosome 17q11.2. The miR-144/451 locus is required for erythroid development and homeostasis. ${ }^{27}$ Both miR-144 and miR-451 are downregulated in bone marrow acute myeloid leukemia cells associated with FLT3-ITD mutation. ${ }^{28} \mathrm{MiR}-451$ is thought to be a potential tumor suppressor that represses MYC and NOTCH1. ${ }^{29}$ Plasma levels of miR-451 are upregulated in breast cancer patients, ${ }^{30}$ similar to what we found in myelodysplastic syndromes patients. The same pattern was also found for another tumor suppressor microRNA, let-7a, which is downregulated in CD34+ bone marrow cells of patients with myelodysplastic syndromes associated with an intermediate or high-risk karyotype, ${ }^{31}$ but is upregulated in plasma. The different profiles of intracellular and extracellular microRNAs may be explained by cellular selection mechanisms or microRNA secretion. ${ }^{32}$ Active secretion of tumor suppressor microRNAs by the stroma may function as a defense mechanism to contain the growth of tumor cells. A direct comparison of these microRNA levels in extracellular circulation and within tumor cells in the same patient will likely provide more definitive answers to these questions.

Among the other microRNAs that were significantly associated with myelodysplastic syndromes in this study, miR-25 is a known regulator of p53 and is involved in stem cell programming. ${ }^{33-35}$ MiR-493 is a tumor suppressor that downregulates RhoC and FZD4, decreases cell motility and migration, and blocks metastatic settlement in bladder and colon cancers. ${ }^{36,37}$ MiR-96 regulates gene expression in bone marrow stem cells, ${ }^{38}$ is overexpressed in human cancer cells, ${ }^{39}$ and is overexpressed in the sera of patients with lung carcinomas. ${ }^{40}$ Plasma levels of a number of other microRNAs were significantly different in patients with myelodysplastic syndromes including miR630, miR-503, miR-651, and miR-655. Mir-630 has been reported to be increased in the blood of patients with lung adenocarcinoma, ${ }^{41}$ and may be involved in p53-regulated cancer cell death. ${ }^{42}$ In our study, we found higher plasma levels of miR-630 in patients with myelodysplastic syndromes as compared with healthy individuals. We also found that miR-503 was upregulated in healthy individuals and in patients with myelodysplastic syndromes with isolated $\operatorname{del}(20 \mathrm{q})$, suggesting this microRNA may be associated with a better prognosis. MiR-503 has been shown to target FGF2 and VEGFA and inhibit tumor angiogenesis and growth. ${ }^{43}$ We showed upregulation of MiR-651 and miR-655 in healthy individuals, but little is known about these microRNAs.

Putting these microRNAs into the context of gene regulatory pathways, microRNAs linked to myelodysplastic syndromes are related to the inflammatory response, cell death and survival, and cancer. Whether the sources of these plasma microRNAs are leukemic cells or stroma cells cannot be determined from this study. Other studies, however, have shown that there is a strong correlation between elevated microRNA levels, such as miR-92a, in plasma and leukemic cells, suggesting an important role in cellular communication. ${ }^{44-46}$

In summary, we performed global microRNA expression profiling on plasma samples from 72 patients with de novo myelodysplastic syndromes and 12 healthy individuals (controls). We identified a number of microRNAs that are potential noninvasive biomarkers for diagnosis and prognostication. Our findings also suggest that there are common pathways that are dysregulated in myelodysplastic syndromes, leukemogenesis, and disease progression. These pathways involve active trafficking of microRNAs between tumor cells and the stromal environment via the extracellular circulation.

\section{Acknowledgments}

We thank Michael Fernandez and John Lee for their excellent technical support of this study. This work was supported, in part, by The University of Texas MD Anderson Cancer Center Faculty Startup Fund 
(to ZZ), The University of Texas MD Anderson Cancer Center Pathology Fellowship Award (to SH), The University of Texas MD Anderson Cancer Center core Grant CA16672 (to CB-R), and by Leukemia SPORE Developmental Research Award (to GAC).

\section{Disclosure/conflict of interest}

The authors declare no conflict of interest.

\section{References}

1 Rollison DE, Howlader N, Smith MT, et al. Epidemiology of myelodysplastic syndromes and chronic myeloproliferative disorders in the United States, 2001-2004, using data from the NAACCR and SEER programs. Blood 2008;112:45-52.

2 Greenberg P, Cox C, LeBeau MM, et al. International scoring system for evaluating prognosis in myelodysplastic syndromes. Blood 1997;89:2079-2088.

3 Brunning RD, Orazi A, Germing U, et al. Myelodysplastic syndromes/neoplasms, overview, In: Swerdlow $\mathrm{SH}$, Campo E, Harris NL, et al. (eds), WHO Classification of Tumours of Haematopoietic and Lymphoid Tissues, 4th edn., IARC: Lyon; 2008, pp 88-93.

4 Kantarjian $\mathrm{H}$, O’Brien S, Ravandi F, et al. Proposal for a new risk model in myelodysplastic syndrome that accounts for events not considered in the original International Prognostic Scoring System. Cancer 2008;113:1351-1361.

5 Schanz J, Tuchler H, Sole F, et al. New comprehensive cytogenetic scoring system for primary myelodysplastic syndromes (MDS) and oligoblastic acute myeloid leukemia after MDS derived from an international database merge. J Clin Oncol 2012;30:820-829.

6 Nybakken GE, Bagg A. The genetic basis and expanding role of molecular analysis in the diagnosis, prognosis, and therapeutic design for myelodysplastic syndromes. J Mol Diagn 2014;16:145-158.

7 Cazzola M, Malcovati L. Myelodysplastic syndromescoping with ineffective hematopoiesis. N Engl J Med 2005;352:536-538.

8 Malcovati L, Germing U, Kuendgen A, et al. Timedependent prognostic scoring system for predicting survival and leukemic evolution in myelodysplastic syndromes. J Clin Oncol 2007;25:3503-3510.

9 Bains A, Luthra R, Medeiros LJ, et al. FLT3 and NPM1 mutations in myelodysplastic syndromes: frequency and potential value for predicting progression to acute myeloid leukemia. Am J Clin Pathol 2011;135: 62-69.

10 Bejar R, Stevenson K, Abdel-Wahab O, et al. Clinical effect of point mutations in myelodysplastic syndromes. N Engl J Med 2011;364:2496-2506.

11 Pellagatti A, Cazzola M, Giagounidis A, et al. Deregulated gene expression pathways in myelodysplastic syndrome hematopoietic stem cells. Leukemia 2010; 24:756-764.

12 Greenberg PL, Tuechler H, Schanz J, et al. Revised international prognostic scoring system for myelodysplastic syndromes. Blood 2012;120:2454-2465.

13 Bartel DP. MicroRNAs: genomics, biogenesis, mechanism, and function. Cell 2004;116:281-297.
14 Croce CM. Causes and consequences of microRNA dysregulation in cancer. Nat Rev Genet 2009;10: 704-714

15 Cortez MA, Bueso-Ramos C, Ferdin J, et al. MicroRNAs in body fluids-the mix of hormones and biomarkers. Nat Rev Clin Oncol 2011;8:467-477.

16 Kosaka N, Iguchi H, Ochiya T. Circulating microRNA in body fluid: a new potential biomarker for cancer diagnosis and prognosis. Cancer Sci 2010;101: 2087-2092.

17 Mitchell PS, Parkin RK, Kroh EM, et al. Circulating microRNAs as stable blood-based markers for cancer detection. Proc Natl Acad Sci USA 2008;105: 10513-10518.

18 Michael A, Bajracharya SD, Yuen PS, et al. Exosomes from human saliva as a source of microRNA biomarkers. Oral Dis 2010;16:34-38.

19 Park NJ, Zhou H, Elashoff D, et al. Salivary microRNA: discovery, characterization, and clinical utility for oral cancer detection. Clin Cancer Res 2009;15:5473-5477.

20 Kosaka N, Izumi H, Sekine K, et al. microRNA as a new immune-regulatory agent in breast milk. Silence 2010;1:7.

21 Zuo Z, Calin GA, de Paula HM, et al. Circulating microRNAs let-7a and miR-16 predict progression-free survival and overall survival in patients with myelodysplastic syndrome. Blood 2011;118:413-415.

22 Johnson WE, Li C, Rabinovic A. Adjusting batch effects in microarray expression data using empirical Bayes methods. Biostatistics 2007;8:118-127.

23 Naqvi K, Garcia-Manero G, Sardesai S, et al. Association of comorbidities with overall survival in myelodysplastic syndrome: development of a prognostic model. J Clin Oncol 2011;29:2240-2246.

24 Garcia-Manero G, Shan J, Faderl S, et al. A prognostic score for patients with lower risk myelodysplastic syndrome. Leukemia 2008;22:538-543.

25 Geiss GK, Bumgarner RE, Birditt B, et al. Direct multiplexed measurement of gene expression with colorcoded probe pairs. Nat Biotechnol 2008;26:317-325.

26 Grasedieck S, Sorrentino A, Langer C, et al. Circulating microRNAs in hematological diseases: principles, challenges, and perspectives. Blood 2013;121: 4977-4984.

27 Rasmussen KD, Simmini S, Abreu-Goodger C, et al. The miR-144/451 locus is required for erythroid homeostasis. J Exp Med 2010;207:1351-1358.

28 Whitman SP, Maharry K, Radmacher MD, et al. FLT3 internal tandem duplication associates with adverse outcome and gene- and microRNA-expression signatures in patients 60 years of age or older with primary cytogenetically normal acute myeloid leukemia: a Cancer and Leukemia Group B study. Blood 2010; 116:3622-3626.

29 Li X, Sanda T, Look AT, et al. Repression of tumor suppressor miR-451 is essential for NOTCH1-induced oncogenesis in T-ALL. J Exp Med 2011;208:663-675.

$30 \mathrm{Ng}$ EK, Li R, Shin VY, et al. Circulating microRNAs as specific biomarkers for breast cancer detection. PLoS One 2013;8:e53141.

31 Vasilatou D, Papageorgiou SG, Kontsioti F, et al. Expression analysis of mir-17-5p, mir-20a and let-7a microRNAs and their target proteins in CD34 + bone marrow cells of patients with myelodysplastic syndromes. Leuk Res 2013;37:251-258.

32 Pigati L, Yaddanapudi SC, Iyengar R, et al. Selective release of microRNA species from normal and 
malignant mammary epithelial cells. PLoS One 2010; 5:e13515.

33 Suh SS, Yoo JY, Nuovo GJ, et al. MicroRNAs/TP53 feedback circuitry in glioblastoma multiforme. Proc Natl Acad Sci USA 2012;109:5316-5321.

34 Kumar M, Lu Z, Takwi AA, et al. Negative regulation of the tumor suppressor p53 gene by microRNAs. Oncogene 2011;30:843-853.

35 Lu D, Davis MP, Abreu-Goodger C, et al. MiR-25 regulates Wwp2 and Fbxw7 and promotes reprogramming of mouse fibroblast cells to iPSCs. PLoS One 2012;7:e40938.

36 Okamoto K, Ishiguro T, Midorikawa Y, et al. miR-493 induction during carcinogenesis blocks metastatic settlement of colon cancer cells in liver. EMBO J 2012;31:1752-1763.

37 Ueno K, Hirata H, Majid S, et al. Tumor suppressor microRNA-493 decreases cell motility and migration ability in human bladder cancer cells by downregulating RhoC and FZD4. Mol Cancer Ther 2012;11:244-253.

38 Laine SK, Alm JJ, Virtanen SP, et al. MicroRNAs miR96, miR-124, and miR-199a regulate gene expression in human bone marrow-derived mesenchymal stem cells. J Cell Biochem 2012;113:2687-2695.

39 Wang Y, Huang JW, Calses P, et al. MiR-96 downregulates REV1 and RAD51 to promote cellular sensitivity to cisplatin and PARP inhibition. Cancer Res 2012;72:4037-4046.

40 Zhu W, Liu X, He J, et al. Overexpression of members of the microRNA-183 family is a risk factor for lung cancer: a case control study. BMC Cancer 2011;11:393.

41 Patnaik SK, Yendamuri S, Kannisto E, et al. MicroRNA expression profiles of whole blood in lung adenocarcinoma. PLoS One 2012;7:e46045.

42 Galluzzi L, Morselli E, Vitale I, et al. miR-181a and miR-630 regulate cisplatin-induced cancer cell death. Cancer Res 2010;70:1793-1803.

43 Zhou B, Ma R, Si W, et al. MicroRNA-503 targets FGF2 and VEGFA and inhibits tumor angiogenesis and growth. Cancer Lett 2013;333:159-169.

44 Tanaka M, Oikawa K, Takanashi M, et al. Downregulation of miR-92 in human plasma is a novel marker for acute leukemia patients. PLoS One 2009;4:e5532.

45 Umezu T, Ohyashiki K, Kuroda M, et al. Leukemia cell to endothelial cell communication via exosomal miRNAs. Oncogene 2013;32:2747-2755.

46 Ohyashiki JH, Umezu T, Kobayashi C, et al. Impact on cell to plasma ratio of miR-92a in patients with acute leukemia: in vivo assessment of cell to plasma ratio of miR-92a. BMC Res Notes 2010;3:347. 